\title{
RFID TECHNOLOGY TO SOLVE TRAFFIC MANAGEMENT PROBLEMS USING INTERNET OF THINGS
}

\author{
Pallavi Gowdoor ${ }^{1}$, Bhavya $\mathrm{S}^{2}$, Rekha $\mathrm{J}^{\mathbf{3}}$, Surendranath $\mathrm{S} \mathrm{K}^{\mathbf{4}}$ \\ ${ }^{I}$ Assistant Professor, Dept. of IS\&E, SKIT, Karnataka, India \\ ${ }^{2}$ B.E student, Dept. of IS\&E, SKIT, Karnataka, India \\ ${ }^{3}$ B.E student, Dept. of IS\&E, SKIT, Karnataka, India \\ ${ }^{4}$ B.E student, Dept. of IS\&E, SKIT, Karnataka, India
}

\begin{abstract}
As each day passes by, the number of vehicles being released to the road is increasing rapidly. The increase in traffic is one of the major causes for the emission of polluted air into the atmosphere. Similarly, the probability of the vehicle meeting with the accident is much more, due to the enormous traffic. Most of such accidents remain undetected and un-notified. The raise in traffic also resulted in the need to monitor the traffic efficiently. An efficient way to control and monitor the air pollution in the emission level, to effectively maintain the traffic in the traffic signals and to detect the accidents and notify them to the respective authorities is being proposed in this system. The proposed system uses the Radio Frequency Identification (RFID) Technology. This technology includes the sensors for detecting the emission of hazardous gases and vehicles met with an accident. The collected data from the sensors is transferred to the inspection system which then notifies the related authorities. The vehicles and traffic signals are interconnected with uniquely identifiable RFID tags with the help of internet that emphasis the concept of Internet of Things (IoT).
\end{abstract}

Keywords: RFID, Internet of Things, emission detection, accident detection, smart traffic signals.

\section{INTRODUCTION}

Everyday millions of people step outside into an environment that has become unsafe for human survival. The major cause for this situation is 'Air Pollution' Diseases like Bronchitis, Emphysema, Mesothelioma, Pneumonia, and Leukemia are a big threat to human life. Air pollution is a huge problem and not just for people living in smog choked cities, through such things as global warming and damage to the ozone layer, it has the potential to affect us all. Road accidents take place in big cities almost every day. Sometimes, these accidents prove to be fatal. Two main factors responsible for road accidents are heavy traffic on roads and rash driving. Pressure on roads has been on increase and the number of vehicles is increasing by leaps and bounds. Traffic congestion is a big problem for everyone within the city. The main reasons why traffic congestion occurs are more cars, poor road management, and poor practices on behalf of employers.

Outside pollution kills millions of people every year, especially in metropolis. In major cities like Delhi, diesel electricity generators and surrounding coal plants have been damaged. To reduce this problem, emission standards have been established. In a typical vehicle exhaust system, there are usually two built in lambda sensors installed on the exhaust pipe that are used to measure the engine ratio $(\lambda)$, which is the stoichiometric value which is normally 1 . These emission standards focus on regulating the pollutants released by automobiles and other powered vehicles. Methods like improvement in vehicle engines or gasoline quality has not solved the emission problem. Traffic
Management leads to various problems like air pollution due to exhaust gas from vehicles, increase in the road accidents and controlling the traffic.

Accidents are one of the leading causes of fatalities. There is no immediate action taken to notify the authorities about the accident which sometimes leads to death of victims. An important indicator of survival rates after an accident is the time between the accident and when emergency medical personnel are dispatched to the accident location. By eliminating the time between when an accident occurs and when the first responders are dispatched to the scene decreases mortality rates, we can save lives. One approach to eliminate the delay after the accident occurrence and first responder dispatch is to use in-vehicle automatic accident detection and notification system, which sense when a traffic accident is occurred and immediately notify the respective authorities.

Nowadays, controlling traffic becomes major issue due to rapid increase of automobiles and also due to large time delays between traffic lights. To overcome these problems, new system is proposed. The system proposed is Wireless Inspection and Notification System (WINS) through the concept of Internet of Things (IoT). This system uses the Radio Frequency Identification (RFID) technology which is a mature wireless communication method. RFID is used to collect and transmit the information of vehicle. In this system, sensors are used to detect the vehicle that emits the exhaust gas and to identify the location when there is accident. Traffic density is also known where sensors are installed. 
In this system, each vehicle has a unique RFID tag for identifying the vehicle. This is sensed by the RFID readers that are installed on the traffic lights. When the vehicle stop in front of red light, reliable reading of emission signals from vehicle can be interrogated. The information of vehicle will be sent to the authorities, so that they can decide which vehicle has to be checked for emission. In the vehicle accident detection system, traffic accidents are automatically detected using the vibration sensors and notification will be sent to the emergency dispatcher after an accident with the help of GPS.

In the traffic controlling system, the density of the road will be sensed by the Ultrasonic sensors which are installed on the road sides. If the distance between the sensor and the vehicle is less, priority will be given to the particular road and the signal will be dismissed for that road. If two roads have more density, either one among them will be given priority. Through these methods, vehicles will be checked regularly for emission. The delay for the notification for the first responder will be reduced when accident occurs. The roads with high density will be given priority to control the traffic.

\subsection{Present Status}

The system includes the $\lambda$-sensors which are implemented only in the high efficiency vehicles. Thus, the vehicles without these sensors do not undergo the emission detection. The accident detection system uses the concept of GSM through the cellular networks for notifying the accidents. It requires a higher stabilized network signal to forward the notifications. In the traffic controller system the time delay tenet is applied to manage the traffic. The system uses Wireless Sensor Network (WSN) which is huge and expensive for practical implementation.

\subsection{Proposed Idea}

The proposed system uses RFID technology to overcome the drawbacks of the present status of the system. RFID is a low-cost and mature wireless communication technology that includes the RFID tags and readers. The vehicle sensor reading can be interrogated along with the corresponding tag ID through a wireless connection among traffic lights and vehicles. The RFID readers are implemented on top of the traffic lights for the interrogation of vehicles that stop in the signals. The RFID tags along with the sensors are placed on the vehicles for the vehicle identification and inspection. The tags when interrogated by the reader with respect to the unique tag ID, the vehicle details that include the vehicle number, owner name and other details along with the sensor values are sent to the reader through the radio frequency signals. The reader then compares the sensed values to the threshold value, if the sensed value exceeds then the data is sent to the IoT through the internet enabled Wi-Fi to notify the respective authority. The ultrasonic sensors are installed on the road side to measure the density of the traffic. The lane with maximum density is given the higher priority.

\section{SYSTEM DESIGN}

\subsection{System Requirements}

Hardware Interfaces:

- Node MCU

- Power supply

- LCD display

- Gas sensor

- Temperature sensor

- Vibration sensor

- Fire sensor

- Ultrasonic sensor

- RFID tag

- RFID reader

- GPS

Software Interfaces:

- Osmosis/Ubidot

- $\quad$ Embedded C Program

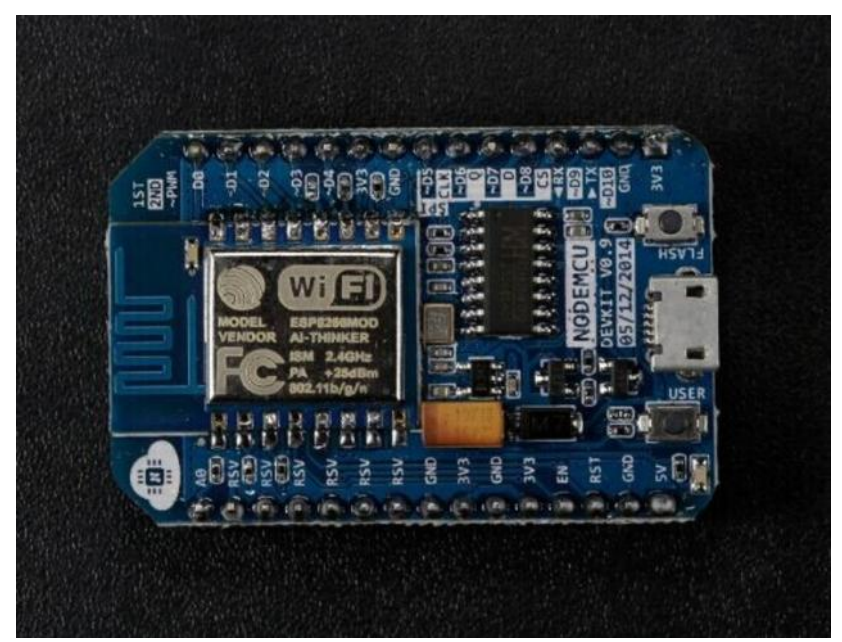

Fig-1: NodeMCU

NodeMCU is an open source IoT platform. It includes the ESP8266 Wi-Fi SoC which is based on the ESP-12 module.

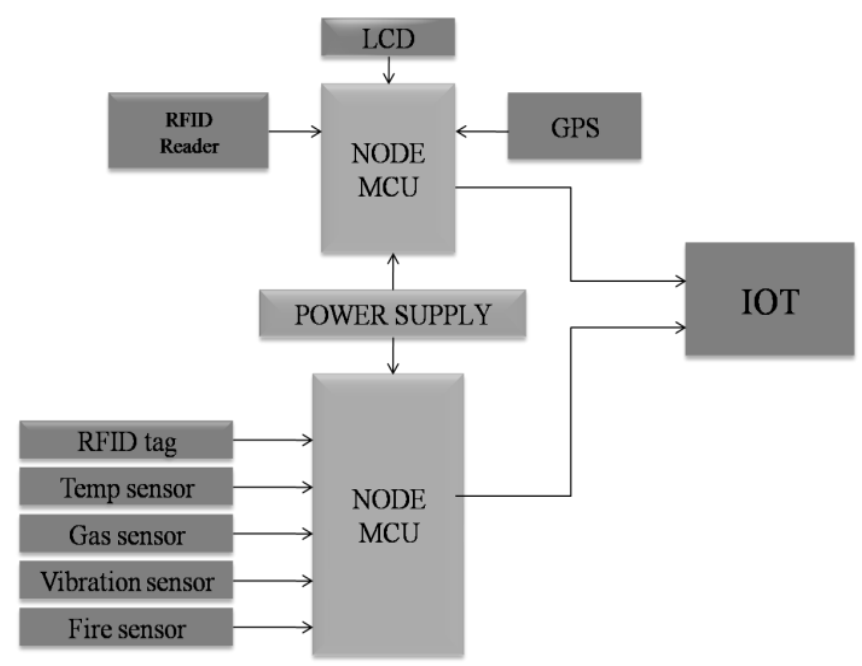

Fig -2: Block diagram 
The above system consists of Node MCU microcontroller interface with sensors (temperature, gas, vibration, fire and ultrasonic sensors). RFID tag is mainly responsible for data collection, while data transport can be realized via RFID reader. GPS is used to locate the accidents. The distance between the RFID reader and the tag is the main interrogation for the traffic controller system. The vibration sensors play the vital role in accident detection system. If the vehicle is met with an accident, notifications are sent to the police department and the nearby hospital for the immediate rescue.

\subsection{Working}

Initially the sensors placed on the vehicle along with the RFID tag senses the gas or the vibration with the help of gas or vibration sensors. When these vehicles enter the range of RFID readers mounted on the traffic signals, they get interrogated with respect to the unique RFID tags. The tag ID with vehicle and owner details along with the sensor values are sent to the RFID reader through the radio frequency signals.

The RFID reader compares the sensor values to the predefined threshold value. If the value exceeds the threshold value then the reader sends the sensor data to the cloud using the Wi-Fi. The IoT platform on cloud then notifies the respective authorities via SMS or e-mail. This is represented in the figure 3 .

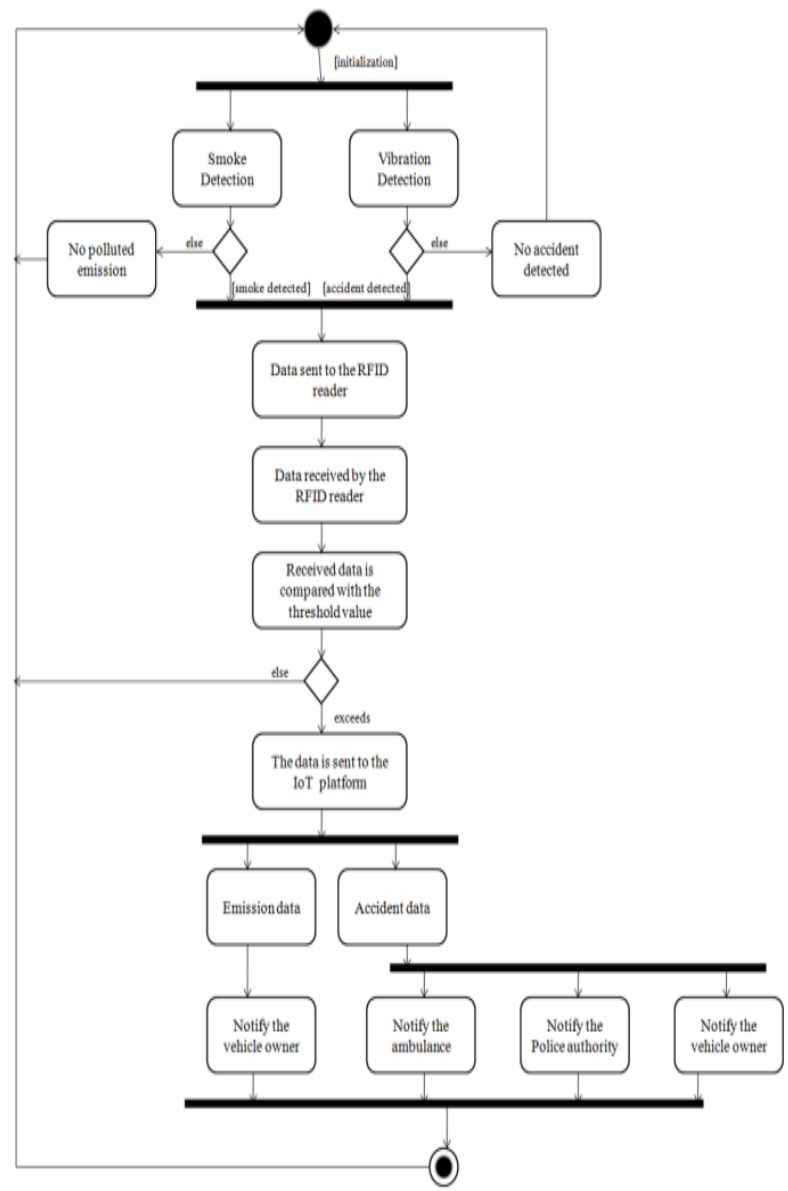

Fig-3: Activity diagram for accident and emission detection
The ultrasonic sensors along with RFID tags are mounted at a specific distance from the RFID readers on the traffic signals. The RFID tags represent the lane on which it is mounted. The sensors measures the distance from the last vehicle. If the distance is lesser, then it indicates that the lane is heavily densed. Thus, it notifies the RFID reader about the density. The RFID reader compares the density of all the lanes and gives priority to heavily densed lane and the signal for that particular lane is released. This is represented in figure 4.

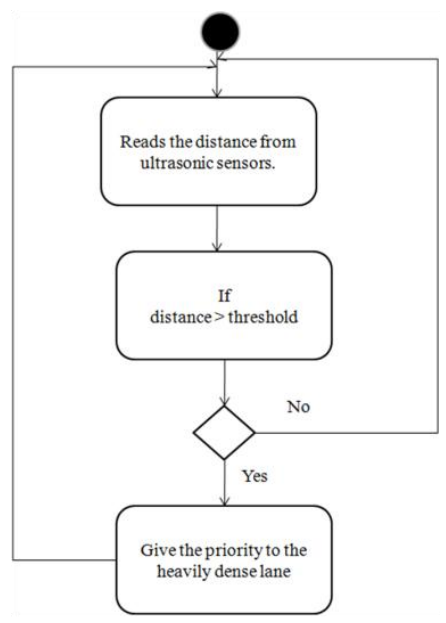

Fig-4: Activity diagram for density based traffic management

\section{IMPLEMENTATION}

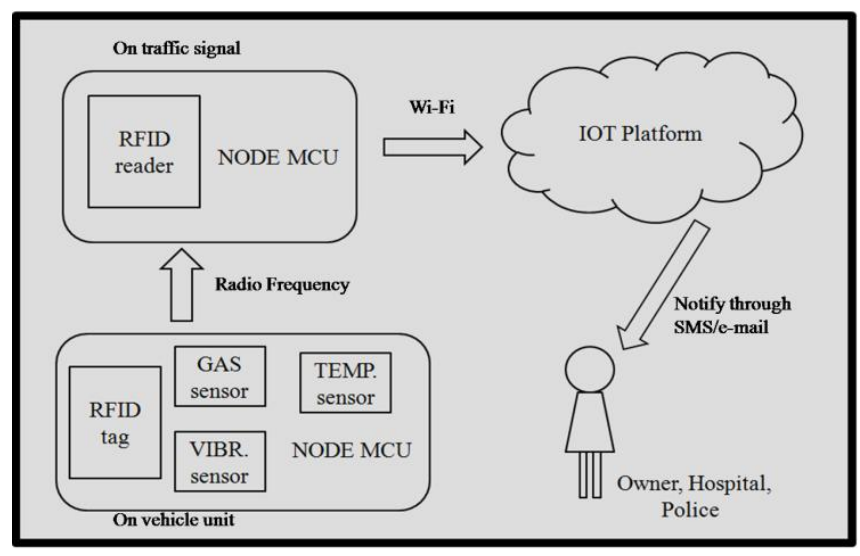

Fig-5: System Architecture.

The system includes three units namely

- On vehicle unit

- On road unit

- On traffic signal unit

The vehicle unit consists of the microcontroller NodeMCU on which the RFID tag, gas, temperature, vibration and the fire sensors are mounted. The vehicle unit is the main component in the accident and the emission detection system. They are responsible in transmitting the sensors values to the reader. 


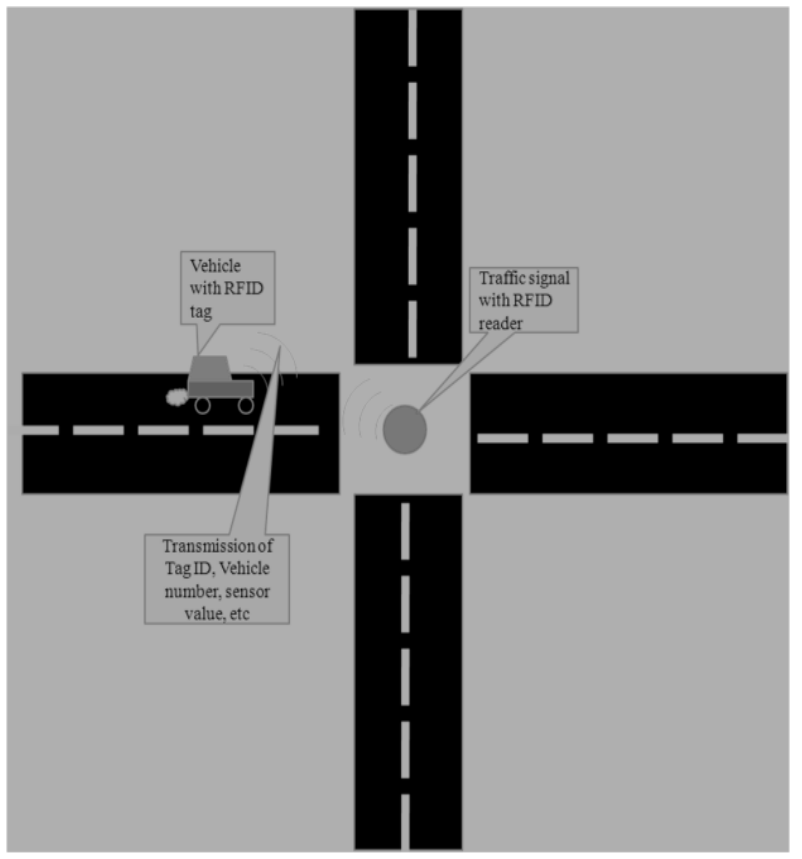

Fig-6: Prototype of emission detection system

The on road unit consists of the microcontroller mounted with the RFID tag and the ultrasonic sensors. It is responsible for determining the density of the traffic and informing the traffic signal unit.

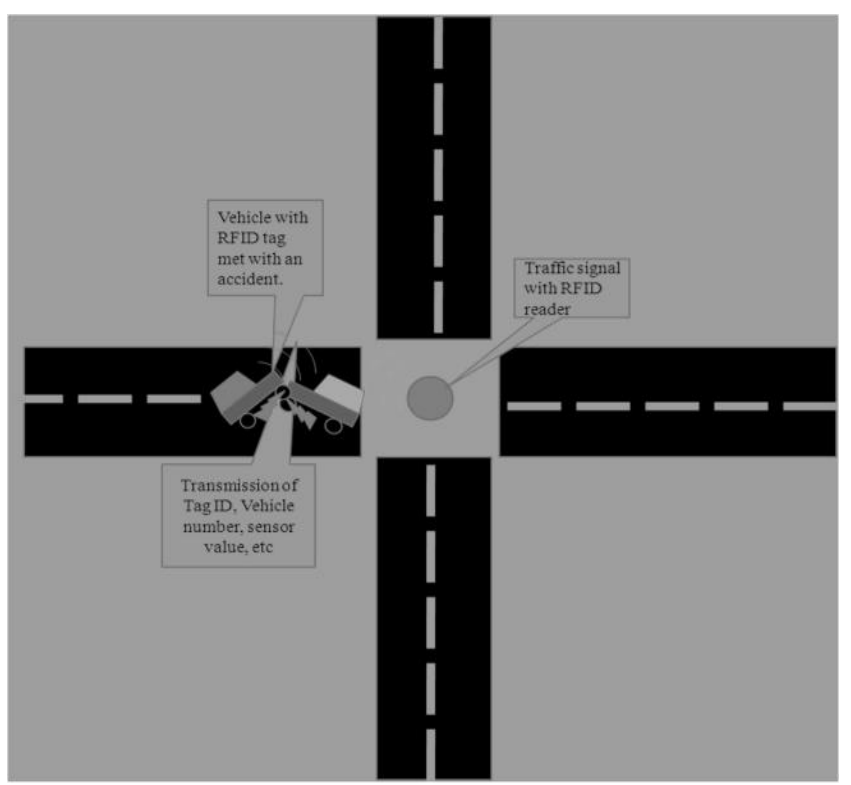

Fig-7: Prototype of accident detection system.

The on traffic signal unit consists of RFID reader mounted on the NodeMCU microcontroller which has inbuilt Wi-Fi module. It is responsible for determining the exceeded sensor data and forwards them to the IoT platform which then notifies to the respective authorities. It is also responsible for the dismiss of signals to the denser lane after comparing all the densities received from the on road unit.

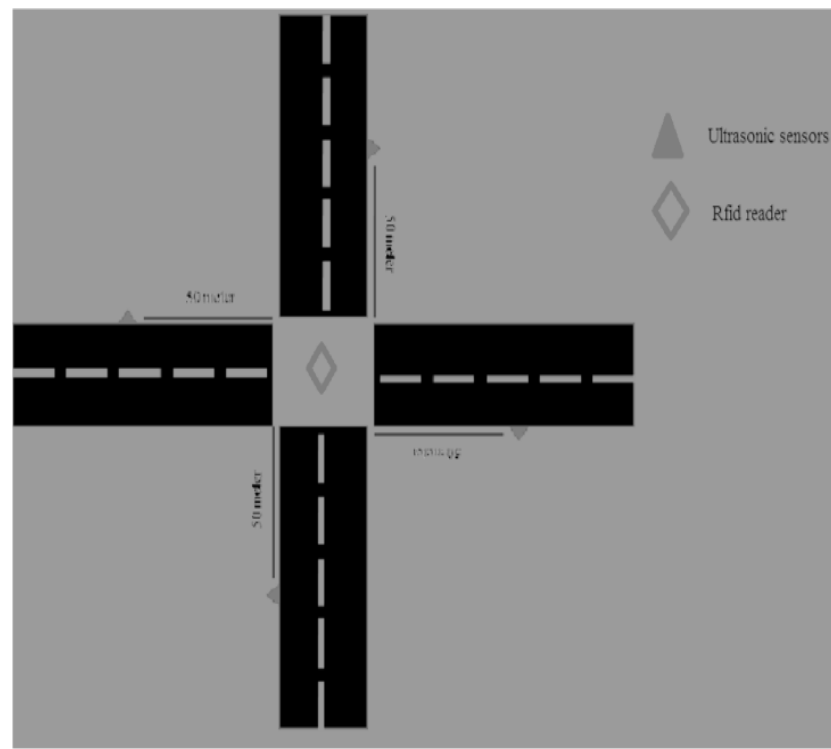

Fig-8: Prototype of density based traffic management.

\section{CONCLUSION}

The proposed system includes the main three features that are primarily required to manage the traffic efficiently. The emission detection helps in control of the air pollution from the emission level of the traffic and also to maintain the engine health of the vehicle. The accident detection aids in the detection of accidents and notifying them using the RFID technology. The time between the accident and when emergency medical personnel are dispatched to the accident location is reduced. The self regulating traffic control system enables the flow of easy traffic in the heavily congested areas.

\section{REFERENCES}

[1] Chi-Man Vong, Pak-Kin Wong, Zi-Qian Ma and KaIn Wang, "Application of RFID Technology and the Maximum Spanning Tee Algorithm for Solving Vehicle Emissions in Cities on Internet of Things", IEEE World Forum on Internet of Things (WF-IoT), 2014.

[2] A. Rajkiran and M. Anusha, "Intelligent Automatic Vehicle Accident Detection System Using Wireless Communication", International Journal of Research Studies in Science, Engineering and Technology, vol. 1, Issue 8, November 2014, pp. 98-101.

[3] R. Pavan Kumar and K V Murali Mohan, "Solving Vehicle Emissions in Cities Based on RFID and GPRS/GSM Technology", International Journal of Research in Advanced Engineering Technologies, vol. 4, Issue 5, 2015.

[4] C-M. Vong, P-K. Wong and W-F. Ip, "Framework of vehicle emission inspection and control through RFID and traffic lights," in Proceedings of 2011 International Conference on System Science and Engineering (ICSSE), 2011, pp. 597-600.

[5] R Udhaya and N Sathish Kumar, "Vehicle Emission Monitoring and Traffic Management", Middle-East Journal of Scientific Research 24 (S1): 268-272, 2016 